\title{
Greater Curvature Antral Flattening: A Radiologic Sign of NSAID-Related Gastropathy
}

\author{
R. L. Laveran-Stiebar, I. Laufer, M. S. Levine \\ Department of Radiology, Hospital of the University of Pennsylvania, 3400 Spruce Street, Philadelphia, PA 19104, USA
}

Received: 10 June 1993/Accepted: 14 July 1993

\begin{abstract}
We have occasionally encountered patients on nonsteroidal antiinflammatory drugs (NSAIDs) in whom double contrast barium studies revealed persistent flattening and stiffening of the distal greater curvature of the stomach. We therefore performed a study to determine the frequency of this finding in patients with NSAID-related gastropathy. Twenty-one cases of erosive gastritis, gastric ulcers, and/or gastric scarring associated with a known history of NSAID use were reviewed by two radiologists who made a joint decision regarding the presence or absence of greater curvature antral flattening. This finding was seen radiographically in five of the 21 patients (24\%). Four of the five patients with antral flattening had associated erosions or ulcers in the gastric antrum. The remaining patient had antral flattening as an isolated finding. Our experience suggests that flattening of the greater curvature of the distal antrum, particularly if associated with erosive gastritis or gastric ulcers, is a useful radiologic sign of NSAIDrelated gastropathy.
\end{abstract}

Key words: Stomach, inflammation-Antral deformity, drug-induced - Gastropathy, NSAID complication.

It is well recognized that nonsteroidal antiinflammatory drugs (NSAIDs) cause mucosal injury in the stomach, with erosions, ulcers, and ulcer scars [1-3]. Over the years, however, we have encountered patients on NSAIDs in whom double-contrast barium studies revealed persistent flattening and straightening of the distal greater curvature of the stomach. To our knowledge, this finding has not been described previously as a sign of NSAID-related gastropathy. We therefore reviewed

Correspondence to: M. S. Levine barium studies in patients on NSAIDs who had gastritis, gastric ulcers, and/or gastric scarring in order to determine the frequency of greater curvature antral flattening in these patients.

\section{Materials and Methods}

A review of our gastrointestinal radiology teaching files revealed 21 patients with a history of recent NSAID use in whom erosive gastritis, gastric ulcers, and/or gastric scarring were diagnosed on double-contrast upper gastrointestinal examinations. The offending agents included aspirin in 13 patients, indomethacin in one, ibuprofen in one, piroxicam in one, and unspecified NSAIDs in five. The films were reviewed by two experienced gastrointestinal radiologists who made a joint decision regarding the presence or absence of greater curvature antral flattening. The appearance and location of associated gastric erosions, ulcers, and ulcer scars were also noted.

\section{Results}

Of the 21 patients, five (24\%) had persistent flattening and straightening of the greater curvature of the distal antrum (Figs. 1-4). This antral flattening was associated with gastric erosions in one patient (Fig. 1), a greater curvature gastric ulcer in one (Fig. 2), and both erosions and ulcers (one greater curvature and one lesser curvature ulcer) in two (Fig. 3). The remaining patient with antral flattening had no evidence of erosions or ulcers (Fig. 4). In two of the three patients with antral flattening and erosions, the erosions had a linear or serpiginous configuration.

\section{Discussion}

It is well recognized that aspirin and other NSAIDs are capable of disrupting the mucosal barrier in the stom- 

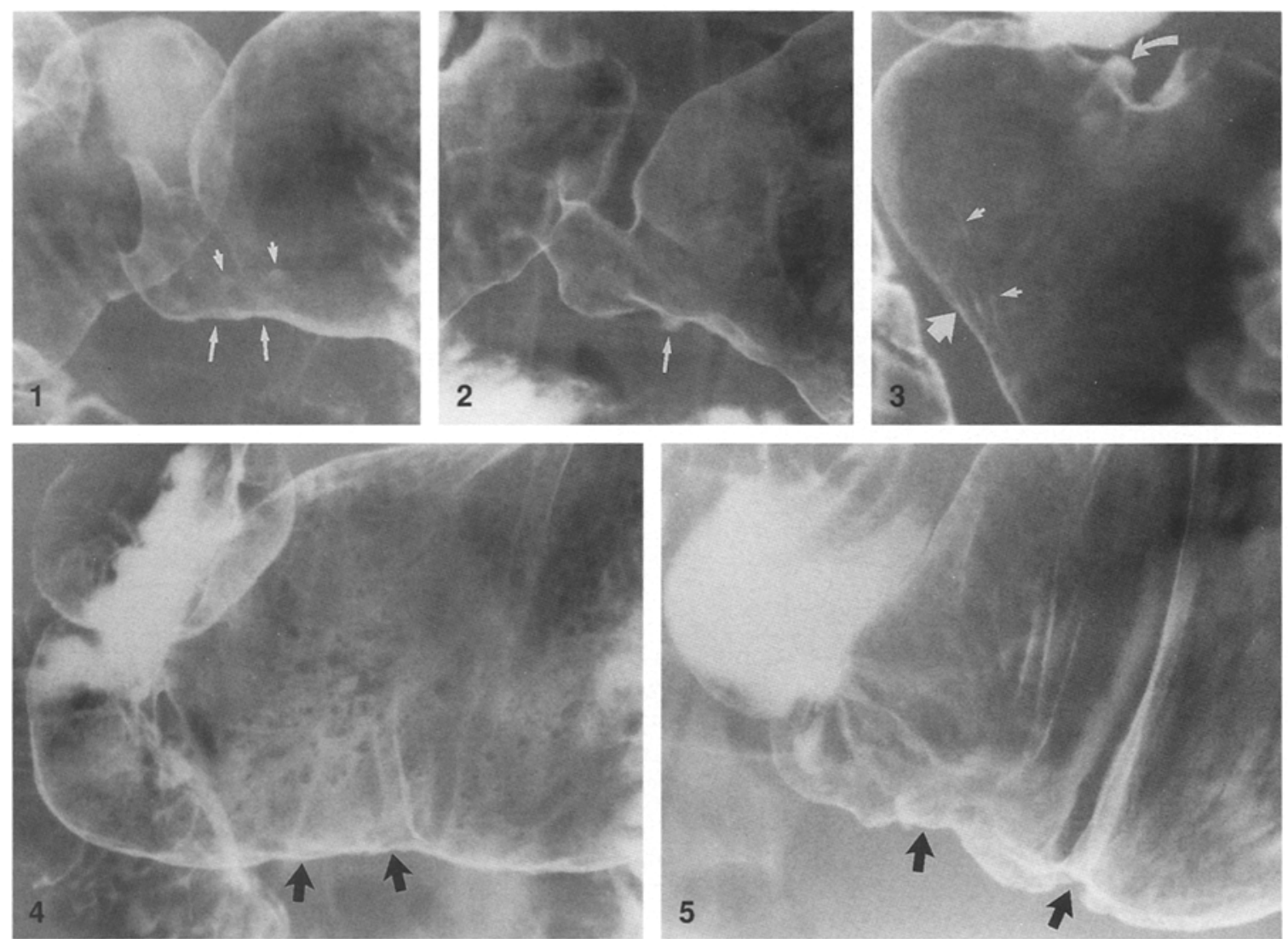

Fig. 1. Antral flattening associated with gastric erosions. A short segment of flattening (large arrows) is seen on the greater curvature of the distal stomach. Several erosions (small arrows) are present in the adjacent antrum.

Fig. 2. Antral flattening associated with a greater curvature ulcer. A small ulcer (arrow) is seen on the greater curvature of the distal antrum with flattening of the adjacent gastric wall.

Fig. 3. Antral flattening associated with linear erosions and a lesser curvature ulcer. A short segment of flattening (large arrow) is seen on the greater curvature of the distal stomach with linear erosions (small arrows) in the adjacent antrum. Also note the presence of a small ulcer (curved arrow) on the lesser curvature of the distal antrum.

Fig. 4. Antral flattening occurring as an isolated finding. A segment of flattening (arrows) is seen on the greater curvature of the antrum. In this case, no erosions or ulcers are seen in the adjacent stomach.

Fig. 5. Greater curvature ulcer scars. Two areas of puckering and deformity (arrows) are seen on the greater curvature of the antrum due to scarring from previous ulcers in these locations. ach, causing erosive gastritis and gastric ulcers [4-10]. This NSAID-related gastropathy may be manifested by a spectrum of findings on double-contrast upper gastrointestinal examinations. Some patients may have erosive gastritis with nonspecific varioliform erosions, but others may have characteristic linear or serpiginous erosions that tend to be clustered on or near the greater curvature of the gastric antrum or body [2]. Still other patients may develop gastric ulcers that often are located on the distal half of the greater curvature $[1,3]$. The frequent location of these erosions and ulcers on the greater curvature is presumably related to the effect of gravity, as the dissolving NSAID tablets or capsules collect in the most dependent portion of the stomach, causing localized mucosal injury in this region [1-3]. In any case, the presence of linear or serpiginous erosions or discrete ulcers on or near the greater curvature of the gastric antrum or body should be highly suggestive of NSAID-related gastropathy.

Our experience indicates that persistent flattening and straightening of the greater curvature of the distal antrum is another radiologic sign of NSAID-related gastropathy (Figs. 1-4). In our study, antral flattening was seen radiographically in five of 21 patients (24\%) with NSAID-related gastric disease. Four of the five patients had associated erosions (often linear or serpiginous) or ulcers in the adjacent antrum (Figs. 1-3). It therefore seems reasonable to postulate that aspirin or other NSAIDs may lead to cycles of recurrent ulceration or erosion formation on or near the greater curvature of the distal stomach and that subsequent scarring may be manifested by flattening and straightening of the greater 
curvature. This antral flattening should be differentiated from gastric ulcer scars associated with radiating folds and puckering or deformity of the adjacent gastric wall (Fig. 5). It should also be differentiated from extrinsic compression of the antrum by an adjacent mass lesion. However, an extrinsic mass usually produces a spherical indentation on the greater curvature rather than flattening and straightening of the gastric wall.

Greater curvature antral flattening is best seen on left posterior oblique views of the stomach. These individuals must be rotated $360^{\circ}$ in order to achieve optimal mucosal coating of the greater curvature [11]. Adequate gaseous distention of the stomach is also essential. If distention is inadequate, subtle areas of wall flattening may not be recognized. Conversely, overdistention of the stomach may distort the gastric contour, preventing recognition of this finding.

In summary, persistent flattening and straightening of the greater curvature of the distal antrum should be recognized as another sign of NSAID-related gastropathy on double-contrast upper gastrointestinal examinations. This antral flattening often is associated with linear or serpiginous erosions or discrete ulcers on or near the greater curvature. Occasionally, however, it occurs as an isolated finding, so that antral flattening may be the only clue to the presence of NSAID-related gastric disease. Detection of this finding should therefore lead to careful questioning of the patient about possible NSAID use.

\section{References}

1. Kottler RE, Tuft RJ. Benign greater curve gastric ulcer: the "sump-ulcer". Br J Radiol 1981;54:651-654

2. Levine MS, Verstandig A, Laufer I. Serpiginous gastric erosions caused by aspirin and other nonsteroidal antiinflammatory drugs. AJR 1986;146:31-34

3. Levine MS, Creteur V, Kressel HY, Laufer I, Herlinger H. Benign gastric ulcers: diagnosis and follow-up with double-contrast radiography. Radiology 1987;164:9-13

4. Davenport HW. Salicylate damage to the gastric mucosal barrier. N Engl J Med 1967;276:1307-1312

5. Thorsen WB, Western D, Tanaka Y, Morrissey JF. Aspirin injury to the gastric mucosa: gastrocamera observations on the effect of pH. Arch Intern Med 1968;121:499-506

6. Cameron AJ. Aspirin and gastric ulcer. Mayo Clin Proc 1975;50:565-570

7. Lanza F, Royer G, Nelson R. An endoscopic evaluation of the effects of non-steroidal anti-inflammatory drugs on the gastric mucosa. Gastrointest Endosc 1975;21:103-105

8. Silvoso GR, Ivey KJ, Butt JH, et al. Incidence of gastric lesions in patients with theumatic disease on chronic aspirin therapy. Ann Intern Med 1979;91:517-520

9. O'Laughlin JC, Hoftiezer JW, Ivey KJ. Effect of aspirin on the human stomach in normals: endoscopic comparison of damage produced one hour, 24 hours, and 2 weeks after administration. Scand J Gastroenterol 1981;16:211-214

10. Lanza FL, Nelson RS, Rack MF. A controlled endoscopic study comparing the toxic effects of sulindac, naproxen, aspirin, and placebo on the gastric mucosa of healthy volunteers. J Clin Pharmacol 1984;24:89-95

11. Levine MS, Rubesin SE, Herlinger H, Laufer I. Double-contrast upper gastrointestinal examination: technique and interpretation. Radiology 1988;168:593-602 